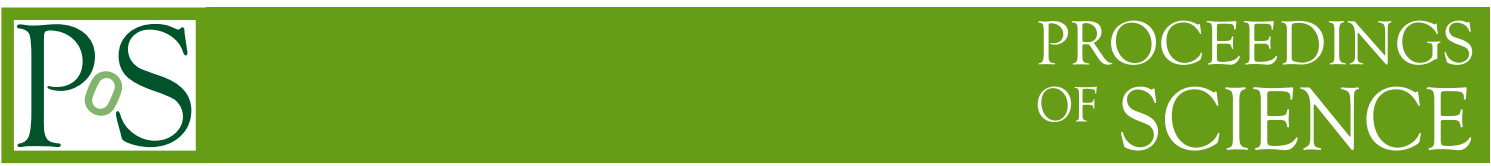

\title{
Neutrino-nucleus scattering at nuSTORM
}

\author{
K. Long* \\ Imperial College London and STFC \\ E-mail: K.Long@Imperial.AC.UK
}

\begin{abstract}
The nuSTORM facility will provide $v_{e}$ and $v_{\mu}$ beams from the decay of low energy muons confined within a storage ring. The instrumentation of the ring, combined with the excellent knowledge of muon decay, will make it possible to determine the neutrino flux at the \%-level or better. The neutrino and anti-neutrino event rates are such that the nuSTORM facility serving a suite of near detectors will be able to measure $v_{e} N$ and $v_{\mu} N$ cross sections with the \%-level precision required to allow the next generation of long-baseline neutrino-oscillation experiments to fulfil their potential. By delivering precise cross-section measurements with a pure weak probe nuSTORM may have the potential to make measurements important to understanding the physics of nucleii. The precise knowledge of the initial neutrino flux also makes it possible to deliver uniquely sensitive sterile-neutrino searches. The concept for the nuSTORM facility will be presented together with an evaluation of its performance. The status of the planned consideration of nuSTORM at CERN in the context of the Physics Beyond Colliders workshop will be summarised. The possibility that nuSTORM could be implemented at the ESS will also be discussed.
\end{abstract}

The 19th International Workshop on Neutrinos from Accelerators-NUFACT2017

25-30 September, 2017

Uppsala University, Uppsala, Sweden

${ }^{*}$ Speaker. 


\section{Introduction}

nuSTORM ("Neutrinos from Stored Muons") is a facility based on a low-energy muon decay ring (see figure 1) [1, 2]. Pions, produced in the bombardment of a target, are captured in a magnetic channel. The magnetic channel is designed to deliver a pion beam with central energy $E_{\pi}$ and energy spread $\sim \pm 20 \% E_{\pi}$ to the muon decay ring. The pion beam is injected into the production straight of the decay ring. Roughly half of the pions decay as the beam passes through the production straight. At the end of the straight, the return arc selects a muon beam of central energy $E_{\mu}<E_{\pi}$ and energy spread $\sim \pm 10 \% E_{\mu}$ that then circulates. Undecayed pions are directed to a beam dump. A detector placed on the axis of the production straight will receive a bright flash of muon neutrinos from pion decay followed by a series of pulses of muon and electron neutrinos from subsequent turns of the muon beam. Appropriate instrumentation in the decay ring and production straight will be capable of determining the integrated neutrino flux with a precision of $\lesssim 1 \%$ [2]. The flavour composition of the neutrino beam from muon decay is known and the neutrino-energy spectrum can be calculated precisely using the Michel parameters and the optics of the muon decay ring. The pion and muon energies $\left(E_{\pi}\right.$ and $\left.E_{\mu}\right)$ can be optimised to:

- Measure $v_{e} A\left(\bar{v}_{e} A\right)$ and $v_{\mu} A\left(\bar{v}_{\mu} A\right)$ interactions with per-cent-level precision; and

- Search for sterile neutrinos with exquisite sensitivity.

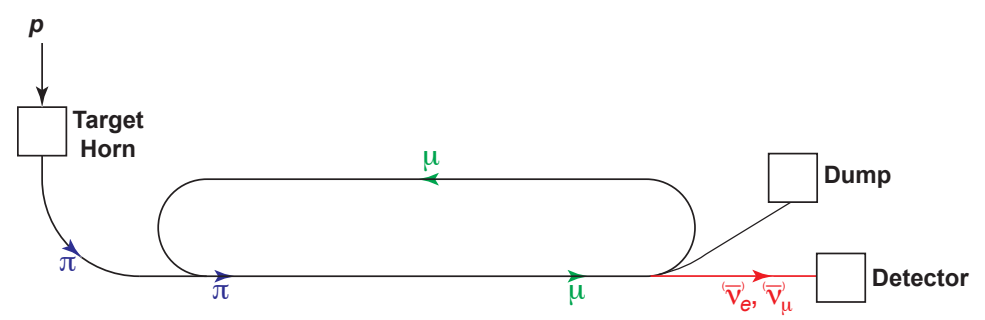

Figure 1: Schematic of the nuSTORM neutrino-beam facility.

\section{Neutrino-nucleus scattering}

The search for CP-invariance violation (CPiV) in present and planned long-baseline neutrinooscillation experiments relies on the measurement of the rate of $v_{e}\left(\bar{v}_{e}\right)$ appearance in $v_{\mu}\left(\bar{v}_{\mu}\right)$ beams. The phenomenological description of the effect relies on the assumption of three neutrinomass eigenstates that mix to produce the three neutrino flavours $[3,4,5,6] . \mathrm{CPiV}$ arises in this framework if the value of a phase parameter, $\delta$, is such that $\sin \delta \neq 0$.

The oscillation probability is a function of the source-detector distance (the baseline) and the neutrino energy. Typical baselines range from $295 \mathrm{~km}$ for T2K [7] and the proposed Hyper-K experiment $[8,9,10,11], 800 \mathrm{~km}$ for NOvA [12] and $1300 \mathrm{~km}$ for the DUNE experiment [13, 14, 15, 16]. Neutrino interactions that occur as the neutrino beam passes through the earth introduce a "matter effect" that causes the oscillation probability of neutrinos to differ from that of antineutrinos. This introduces an "apparent" CPiV effect that depends on the neutrino mass hierarchy. 
The discovery of $\mathrm{CPiV}$ in neutrino oscillations requires that the "true" CPiV that depends on $\delta$ be distinguished from the apparent $\mathrm{CPiV}$ that arises from neutrino interactions with the earth.

The projected sensitivity to CPiV of the DUNE experiment is plotted as a function of exposure in figure 2 [14]. An exposure of $288 \mathrm{kt} \mathrm{MW}$ years will be achieved after seven years of running, with the planned staging to reach a total detector mass of $40 \mathrm{kt}$ and a proton beam-power of $1.2 \mathrm{MW}$ [17]. Equal exposures in neutrino and antineutrino mode have been assumed. The DUNE collaboration presents the sensitivity as a function of the assumed normalisation uncertainties on the $v_{e}$ and $\bar{v}_{e}$ appearance signals. Reducing the $\bar{v}_{e}$ normalisation uncertainty from $3 \%$ to $1 \%$ brings the exposure required to exclude CP invariance at the $3 \sigma$ confidence level over $75 \%$ of all possible values of $\delta$ down from $\sim 1200 \mathrm{kt} \mathrm{MW}$ years to $\sim 600 \mathrm{kt}$ MW years.
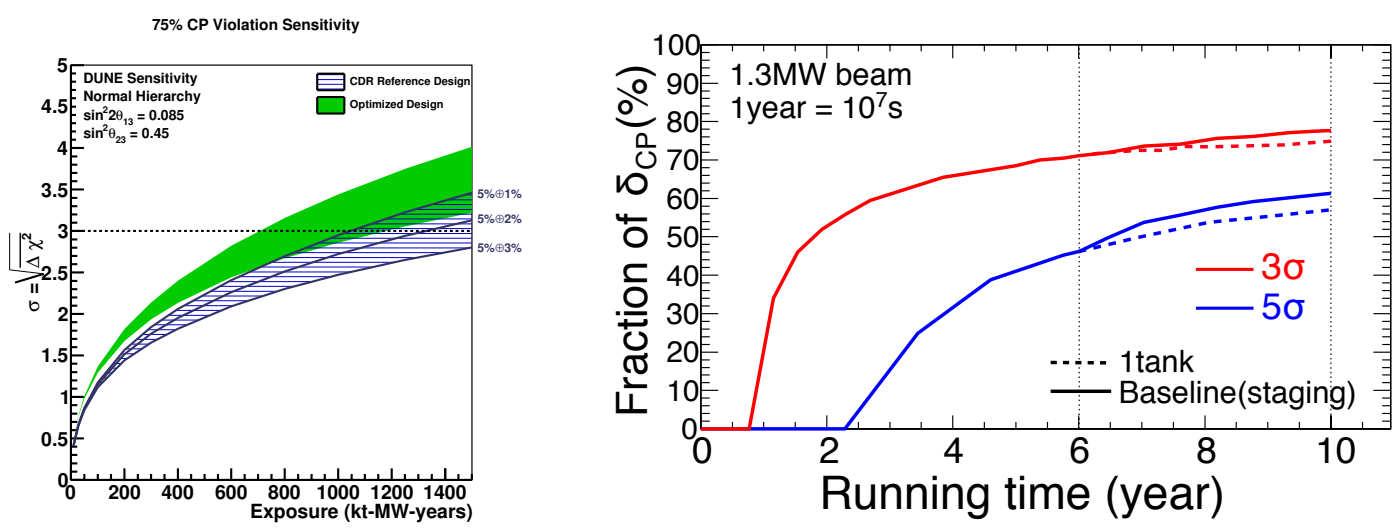

Figure 2: Left panel: Expected sensitivity of the DUNE experiment to CP-invariance violation plotted as a function of exposure in $\mathrm{kt} \cdot \mathrm{MW} \cdot \mathrm{years}$ assuming equal running in neutrino and antineutrino mode, for a range of values for the $v_{e}$ and $\bar{v}_{e}$ signal-normalisation uncertainties (from $5 \%$ to $1 \%$ ) added in quadrature to an uncertainty of $5 \%$ on the normalisation of the background. The sensitivities shown are for the exclusion of CP-invariance conservation over $75 \%$ of the available range of values of $\delta$ assuming the normal hierarchy. The two bands represent a range of potential beam designs: the blue hashed band is for the CDR Reference Design and the solid green band is for the optimised design. The figure is taken from [14]. Right panel: fraction of all values of the CPiV phase, $\delta\left(=\delta_{\mathrm{CP}}\right)$, for which $\delta=\delta_{\mathrm{CP}}=0, \pi$ can be excluded at $3 \sigma$ (red) or $5 \sigma$ (blue) plotted as a function of running time. An exposure of $13 \mathrm{MW} \times 10^{7} \mathrm{~s}$ is expected to be achieved after 10 years of operation. Figure updated from [9].

The projected sensitivity of the Hyper-K experiment, updated from [9], is also shown in figure 2. An exposure of $13 \mathrm{MW} \times 10^{7} \mathrm{~s}$ will be achieved after ten years assuming a 1:3 ratio between neutrino and anti-neutrino running. The planned staged implementation of two $187 \mathrm{kt}$ detectors is indicated, a proton beam-power of $1.3 \mathrm{MW}$ at $30 \mathrm{GeV}$ has been assumed. The systematic uncertainties assumed by the Hyper-K collaboration in their estimation of the CPiV sensitivity of their experiment dominated by the combined "flux and near-detector" and the "cross-section model" uncertainties [9].

In addition to systematic uncertainties, a lack of knowledge of $v_{e, \mu} A\left(\bar{v}_{e, \mu} A\right)$ cross sections or inaccuracies in the simulation of the hadronic final state can lead to biases in the parameters extracted from the data. Such biases may arise, for example, from mis-classification of events [18] or mis-reconstruction of the energy of the incident neutrino [19, 20, 21, 22]. A discussion of 
possible sources of bias is presented in [23]. When searching for CPiV, any effect that differs in $v_{e} A$ and $\bar{v}_{e} A$ scattering and is not quantitatively understood is particularly pernicious since such a difference may be mis-interpretted as a signal for CPiV.

The next generation of long-baseline neutrino-oscillation experiments, DUNE and Hyper-K have the potential to observe CPiV violation. To maximise the scientific impact of the large, exquisitely-precise data sets that they will collect requires that the $v_{e}\left(\bar{v}_{e}\right)$ and $v_{\mu}\left(\bar{v}_{\mu}\right)$ cross sections be known with percent-level precision and that uncertainties associated with cross-section models are also under control at the percent level.

\section{3. nuSTORM and the CERN Physics Beyond Colliders study}

A detailed study of nuSTORM that includes consideration of the implementation of the facility at FNAL may be found in $[2,24,25]$. A preliminary investigation of possible options for siting the facility at CERN may be found in [26, 27]. In these studies the facility was optimised for the search for a light sterile neutrino.

In September 2016 CERN established the Physics Beyond Colliders (PBC) study group to consider ways in which the accelerators at CERN could be used or extended to support a diverse physics programme to complement the energy-frontier physics being pursued using the LHC [28]. The feasibility of implementing nuSTORM at CERN was included as a work package in the PBC study. Within the PBC context, the scientific objectives of nuSTORM are:

- To make detailed and precise measurements of neutrino-nucleus interactions not only as a service to the long- and short-baseline neutrino oscillation programmes but also as a means of studying the nucleus using a weak probe and seeking evidence for non-standard interactions; and

- To take forward the search for light sterile neutrinos should the results of the Short Baseline Neutrino (SBN) programme at FNAL [29] indicate that such a programme is required.

The potential for nuSTORM to establish a new technique for the study of fundamental particles and their interactions is recognised. The PBC study does not include consideration of a possible six-dimensional-cooling experiment (see for example [30]) to follow the demonstration of the reduction of normalised transverse emittance that will be provided by the international Muon Ionization Cooling Experiment (MICE) [31].

The sensitivity of all oscillation experiments depends on the precision of models of the structure of the nucleus and the details of simulations of neutrino scattering. nuSTORM will provide a beam of precisely known flavour for which the flux will be known to $1 \%$ or better. Instrumented with a detector capable of making measurements of exclusive final states and of reconstructing $Q^{2}$ and $W$, nuSTORM can deliver the data required for precise models of the nucleus and of neutrinonucleus scattering to be developed if the neutrino-beam energy spans the range $1 \lesssim E_{v} \lesssim 6 \mathrm{GeV}$. These considerations lead to the following specification for the energy of the circulating muon beam:

- Maximum stored muon energy, $E_{\mu}=6 \mathrm{GeV}$; and 
- It must be possible to vary the muon-beam energy in the range $1 \lesssim E_{\mu} \lesssim 6 \mathrm{GeV}$.

Since the neutrino-energy spectrum is precisely known once the muon-beam energy is specified, the falling edge of the neutrino-energy spectrum can be used to calibrate the energy response of the neutrino detectors. Further, by combining data taken with different stored-muon energies, as described for NuPRISM in [32], cross sections may be determined in narrow neutrino-energy bands.

A sketch of the proposed siting of nuSTORM at CERN is shown in figure 3. The existing fast-extraction from the SPS that serves beam to the West Area will be exploited. Protons will be extracted into a new transfer line and delivered to a target/capture system that will select $\sim$ $8 \mathrm{GeV}$ pions and transport them to the decay ring. The design of the decay ring will be revised to accommodate the maximum muon-beam energy of $6 \mathrm{GeV}$ and to provide the ability to store muon beams with energies in the range $1 \lesssim E_{\mu} \lesssim 6 \mathrm{GeV}$. The feasibility study will take into account the number of protons on target required and the extracted-beam parameters and will consider the fast extraction from the SPS and the proton-beam transport to the pion-production target. Preliminary investigations of the engineering of the target, horn, target complex and proton-beam absorber will be carried out. An important aspect of the feasibility study will be consideration of the radiationprotection issues raised by the target and the pion and muon fluxes.

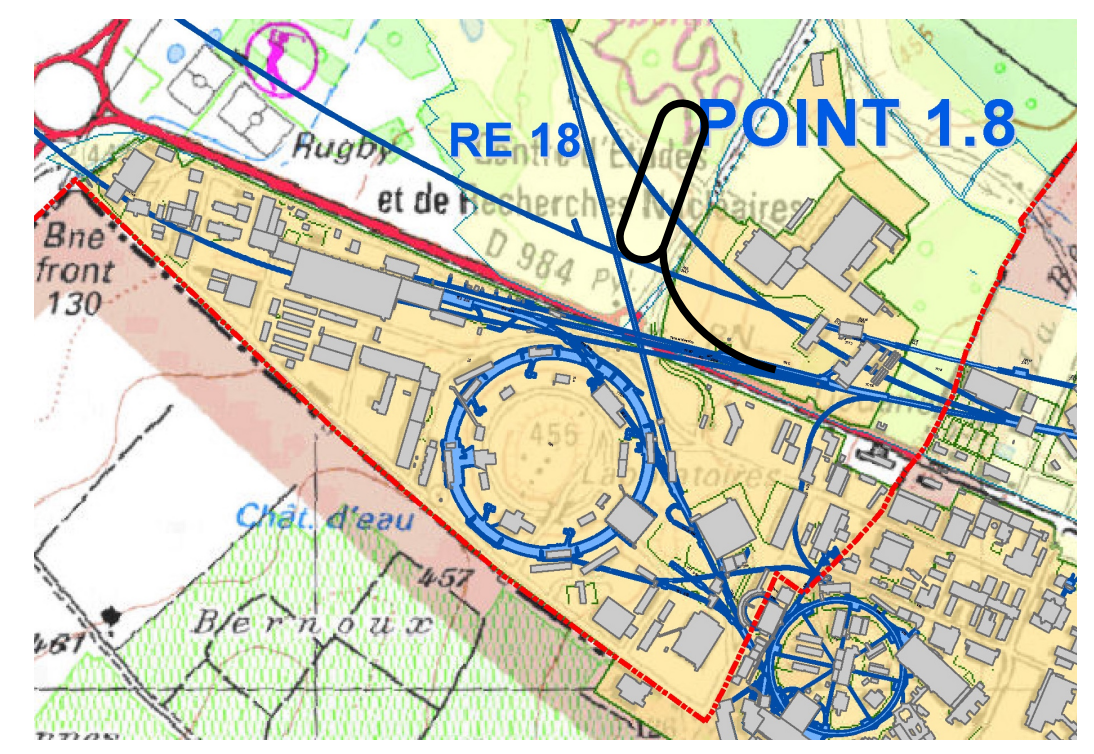

Figure 3: Preliminary sketch of the proposed implementation of nuSTORM at CERN. The blue solid lines show the existing tunnels and transfer lines serving the West Area at CERN. The proposed transfer line to serve nuSTORM and the nuSTORM storage ring location are indicated by the black solid lines. (I. Efthimiopoulos, private communication.)

The potential of the cross-section-measurement programme was evaluated in [2] assuming a neutrino detector with the performance of the HiResM $v$ detector [33]. This analysis showed that nuSTORM has the potential to improve the systematic uncertainty on muon-neutrino (muon-antineutrino) CCQE cross section measurements by a factor of $\sim 5-6$. The $v_{e} A\left(\bar{v}_{e} A\right)$ cross-section measurements that can be made with nuSTORM will be unique. 


\section{4. nuSTORM as a possible upgrade to the ESS}

A possible upgrade of the ESS accelerator complex to serve a programme of neutrino physics is being discussed $[34,35]$ (see figure 4 ). To deliver a very high power proton beam onto a pionproduction target it is proposed to inject many proton-bunch trains into an accumulator ring. Fast extraction of the accumulated protons onto a target/horn-focusing system will generate an intense low-energy neutrino beam. The low neutrino-beam energy will be exploited to study electronneutrino appearance at the second-oscillation maximum to search for CPiV. This facility can be developed to serve nuSTORM by boosting the proton beam energy, delivering the beam to an appropriate target/pion-capture system and subsequently injecting the captured pion beam into the nuSTORM decay ring. The proposed set-up is sketched in figure 4.
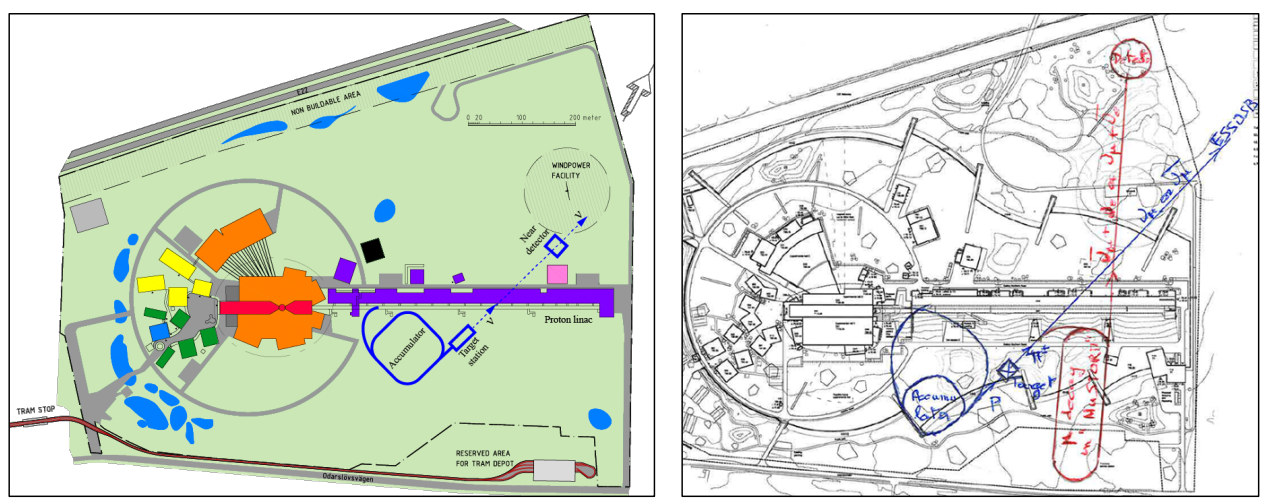

Figure 4: Left panel: schematic diagram of the ESS Neutrino Super Beam [34, 35]. Extraction from the ESS linac, transport and injection into an accumulator ring is shown together with the proposed location of the pion production target. Right panel: sketch of a possible implementation of nuSTORM at ESS based on a ring to boost the proton-beam energy such that the nuSTORM pion-beam energy can be created. The proposed location of the nuSTORM storage ring is also shown. (T. Ekelöf, private communication.)

\section{Conclusions}

The Neutrinos from Stored Muons (nuSTORM) facility is capable of delivering measurements of $v_{e, \mu} N\left(\bar{v}_{e, \mu} N\right)$ scattering for which the flux uncertainty can be reduced to $1 \%$ or better. Such measurements will reduce the systematic uncertainties and biases in future long-baseline neutrinooscillation experiments thereby enhancing their sensitivity to leptonic CP-invariance violation and improving the precision of their measurements of the oscillation parameters. The cross-section measurement programme at nuSTORM has the potential to contribute to the understanding of nuclear physics through the use of a pure weak probe that is $100 \%$ polarised. Should the results of the FNAL Short Baseline Neutrino Program indicate that future searches for light sterile neutrinos are required, nuSTORM can support an exquisitely sensitive sterile-neutrino search programme.

The implementation of nuSTORM at CERN is being studied within the Physics Beyond Colliders Study Group. The goals of the study are to provide a preliminary proposal for siting the facility at CERN, to re-optimise the facility for the neutrino-scattering programme and to demonstrate through simulation that the normalisation of the neutrino flux can be constrained to $\lesssim 1 \%$. 
The programme described here will establish nuSTORM as an option for CERN in time for the next update of the European Strategy for Particle Physics. nuSTORM can be implemented at the ESS by developing the accelerator complex proposed for the ESS Neutrino Super Beam.

\section{References}

[1] NUSTORM COLLABORATION collaboration, P. Kyberd et al., nuSTORM - Neutrinos from STORed Muons: Letter of Intent to the Fermilab Physics Advisory Committee, 1206.0294.

[2] NUSTORM collaboration, D. Adey et al., nuSTORM - Neutrinos from STORed Muons: Proposal to the Fermilab PAC, tech. rep., Fermi National Accelerator Laboratory, 2013.

[3] B. Pontecorvo, Inverse beta processes and nonconservation of lepton charge, Sov. Phys. JETP 7 (1958) 172-173.

[4] B. Pontecorvo, Neutrino experiments and the question of leptonic-charge conservation, Sov. Phys. JETP 26 (1968) 984-988.

[5] Z. Maki, M. Nakagawa and S. Sakata, Remarks on the unified model of elementary particles, Prog. Theor. Phys. 28 (1962) 870-880.

[6] Particle Data Group collaboration, C. Patrignani et al., Review of Particle Physics, Chin. Phys. C40 (2016) 100001.

[7] The T2K collaboration, "T2K." http://t2k-experiment.org, 2013.

[8] Hyper-Kamiokande Working Group collaboration, K. Abe et al., A Long Baseline Neutrino Oscillation Experiment Using J-PARC Neutrino Beam and Hyper-Kamiokande, 1412. 4673.

[9] Hyper-Kamiokande Proto-Collaboration collaboration, K. Abe et al., Physics potential of a long-baseline neutrino oscillation experiment using a J-PARC neutrino beam and Hyper-Kamiokande, PTEP 2015 (2015) 053C02, [1502. 05199].

[10] HyPER-KAMIOKANDE collaboration, Hyper-Kamiokande Design Report, .

[11] Hyper-Kamiokande Proto- collaboration, K. Abe et al., Physics Potentials with the Second Hyper-Kamiokande Detector in Korea, 1611.06118.

[12] The NOvA collaboration, "NOvA Neutrino Experiment." https://www-nova.fnal.gov, 2016.

[13] DUNE collaboration, R. Acciarri et al., Long-Baseline Neutrino Facility (LBNF) and Deep Underground Neutrino Experiment (DUNE), 1601.05471.

[14] DUNE collaboration, R. Acciarri et al., Long-Baseline Neutrino Facility (LBNF) and Deep Underground Neutrino Experiment (DUNE), 1512.06148.

[15] DUNE collaboration, J. Strait et al., Long-Baseline Neutrino Facility (LBNF) and Deep Underground Neutrino Experiment (DUNE), 1601.05823.

[16] DUNE collaboration, R. Acciarri et al., Long-Baseline Neutrino Facility (LBNF) and Deep Underground Neutrino Experiment (DUNE), 1601.02984.

[17] J. Cao et al., Roadmap for the international, accelerator-based neutrino programme, 1704.08181.

[18] P. Coloma and P. Huber, Impact of nuclear effects on the extraction of neutrino oscillation parameters, Phys. Rev. Lett. 111 (2013) 221802, [1307.1243]. 
[19] P. Coloma, P. Huber, C.-M. Jen and C. Mariani, Neutrino-nucleus interaction models and their impact on oscillation analyses, Phys. Rev. D89 (2014) 073015, [1311.4506].

[20] A. M. Ankowski, P. Coloma, P. Huber, C. Mariani and E. Vagnoni, Missing energy and the measurement of the CP-violating phase in neutrino oscillations, Phys. Rev. D92 (2015) 091301, [1507.08561].

[21] M. Ankowski, A., O. Benhar, P. Coloma, P. Huber, C. M. Jen, C. Mariani et al., Neutrino energy reconstruction in disappearance experiments with calorimetric and kinematic methods, Nuovo Cim. C39 (2016) 233.

[22] A. M. Ankowski, Nuclear effects are relevant to the calorimetric reconstruction of neutrino energy, 1704.07835.

[23] A. M. Ankowski and C. Mariani, Systematic uncertainties in long-baseline neutrino-oscillation experiments, J. Phys. G44 (2017) 054001, [1609.00258].

[24] T. Lackowski, S. Dixon, R. Jedziniak, M. Blewitt and L. Fink, nuSTORM Project Definition Report, tech. rep., Fermi National Accelerator Laboratory, 2013.

[25] P. Kyberd et al., nustorm costing document, May, 2013.

[26] D. Adey, C. Ankenbrandt, S. Agarwalla, R. Asfandiyarov, J. Back, G. Barker et al., Neutrinos from stored muons (storm): Expression of interest, Tech. Rep. CERN-SPSC-2013-015. SPSC-EOI-009, CERN, Geneva, Apr, 2013.

[27] D. Adey, S. Agarwalla, C. Ankenbrandt, R. Asfandiyarov, J. Back et al., Neutrinos from Stored Muons nuSTORM: Expression of Interest, 1305.1419.

[28] Jaeckel, J. and Lamont. M. and Vallee, C., "Physics Beyond Colliders.” http://pbc.web.cern.ch, 2016.

[29] The Short Baseline Neutrino Program, "The Short Baseline Neutrino Program.” https://web.fnal.gov/collaboration/sbn/_layouts/15/start.aspx\#/SitePages/Home.aspx, 2014.

[30] D. Stratakis, Six-Dimensional Ionization Cooling for Muon Accelerators with Vacuum RF Technology, ICFA Beam Dyn. Newslett. 65 (2014) 54-62.

[31] The MICE collaboration, "INTERNATIONAL MUON IONIZATION COOLING EXPERIMENT." http://mice.iit.edu.

[32] M. Wilking, E61: The j-parc intermediate water cherenkov detector, 2017.

[33] S. Mishra, Precision measurements and a high resolution near detector for Long-Baseline Neutrino program, Prog.Part.Nucl.Phys. 64 (2010) 202-204.

[34] ESSnuSB Collaboration collaboration, E. Baussan et al., A Very Intense Neutrino Super Beam Experiment for Leptonic CP Violation Discovery based on the European Spallation Source Linac: A Snowmass 2013 White Paper, 1309.7022.

[35] ESSNUSB collaboration, E. Wildner, ESSnuSB: a Project for Leptonic CP Violation Discovery based on the European Spallation Source Linac, Nucl. Part. Phys. Proc. 265-266 (2015) 195-197. 